\title{
Efficient and Secure Fingerprint Verification for Embedded Devices
}

\author{
Shenglin Yang, ${ }^{1}$ Kazuo Sakiyama, ${ }^{2}$ and Ingrid Verbauwhede ${ }^{2}$ \\ ${ }^{1}$ Department of Electrical Engineering, University of California, Los Angeles, CA 90095, USA \\ ${ }^{2}$ ESAT-COSIC, Katholieke Universiteit Leuven, Kasteelpark Arenberg 10, 3001 Leuven-Heverlee, Belgium
}

Received 9 March 2005; Revised 22 September 2005; Accepted 21 January 2006

Recommended for Publication by Roger Woods

\begin{abstract}
This paper describes a secure and memory-efficient embedded fingerprint verification system. It shows how a fingerprint verification module originally developed to run on a workstation can be transformed and optimized in a systematic way to run real-time on an embedded device with limited memory and computation power. A complete fingerprint recognition module is a complex application that requires in the order of $1000 \mathrm{M}$ unoptimized floating-point instruction cycles. The goal is to run both the minutiae extraction and the matching engines on a small embedded processor, in our case a $50 \mathrm{MHz} \mathrm{LEON}-2$ softcore. It does require optimization and acceleration techniques at each design step. In order to speed up the fingerprint signal processing phase, we propose acceleration techniques at the algorithm level, at the software level to reduce the execution cycle number, and at the hardware level to distribute the system work load. Thirdly, a memory trace map-based memory reduction strategy is used for lowering the system memory requirement. Lastly, at the hardware level, it requires the development of specialized coprocessors. As results of these optimizations, we achieve a $65 \%$ reduction on the execution time and a $67 \%$ reduction on the memory storage requirement for the minutiae extraction process, compared against the reference implementation. The complete operation, that is, fingerprint capture, feature extraction, and matching, can be done in real-time of less than 4 seconds.
\end{abstract}

Copyright (c) 2006 Shenglin Yang et al. This is an open access article distributed under the Creative Commons Attribution License, which permits unrestricted use, distribution, and reproduction in any medium, provided the original work is properly cited.

\section{INTRODUCTION}

Biometric verification systems offer great security and convenience due to the uniqueness and efficiency of the personal biometric information. However, one of the most significant disadvantages of these systems is that the biometric information cannot be easily recalled. For example, in a fingerprint authentication application, once the finger used as a password is compromised, it never can be used again. In a traditional biometric recognition system, the biometric template is usually stored on a central server during enrollment. The candidate biometric signal captured by the front-end input device is sent to the server where the processing and matching steps are performed. In this case, the safety of the precious biometric information cannot be guaranteed because attacks might occur during the transmission or on the server. Some embedded fingerprint verification systems try to decentralize the storage of the information by storing the fingerprint template into a device such as a smart card [1]. Although this provides higher security for the fingerprint matching process as well as the template storage, the minutiae extraction process still runs outside on the card reader and the transmission of the input fingerprint information still can lead to the disclosure of the important biometric data. What is unique in our proposed method is that both the minutiae extraction and the matching process are executed locally on the embedded device, gaining maximum security of the system. The embedded device has limited computation resource and memory space. This requires that the signal processing procedure must be fast and compact. Therefore, the goal of our work is to show that efficient minutia extraction modules can be realized in the context of an embedded device. It does require a systematic approach that looks at different abstraction levels to reach this goal.

Different fingerprint authentication applications might use the same fingerprint due to the limited number of fingers for one person. So the fingerprints stolen from one application could also be used in some other applications [2]. Therefore the secure storage of the fingerprint template is becoming extremely important. By extracting the minutiae and performing the matching locally, the system can avoid attacks on the communication and the server. Also it avoids 
the need for biometric data to be stored on multiple servers for multiple applications. One alternative is to encrypt the sensitive data before it leaves the embedded device. Then an attack on the link is not possible. This is certainly an option for some applications. There are two main reasons why we opted to process the biometrics on the embedded device. The first one is of perceived privacy. In our proposed system, the fingerprint template needs to be stored only once and the user keeps it with him. We want to avoid that biometric data is stored in multiple places with different levels of security. For example, it could be used to enter nuclear facilities as well as the locker room of the local sports club. If the data is sent over to be processed elsewhere, the user has to trust that his/her personal data is treated confidentially and not disclosed. The second reason is that in the future, we envision that most embedded devices are connected with a wireless link. The radio transmission energy is a much larger cost than the local processing energy [3]. This can be orders of magnitude in battery-operated devices. Thus the trend in embedded devices is to minimize the amount of data that needs to be transmitted. However, it is still possible to compromise the plain storage of the template in an embedded device. To improve the security of the storage, we propose a secure matching algorithm based on a well-defined transformed template structure, which does not contain the original fingerprint information.

The design of the embedded verification requires optimizations at each design step. At the algorithm level, the secure matching algorithm has been developed to address security issues in embedded devices. At the software level, optimization based on profiling results reduces the required system cycle number. At the hardware level, optimizations are performed at both the memory organization and the datapath acceleration. A memory trace map-based memory reduction strategy is applied to lower the system memory requirements. Memory-mapped techniques have been used to design the acceleration coprocessors.

The contributions of this paper are: (1) high-speed optimization technique using the pattern characteristics of the fingerprints; (2) DFT accelerator by creating dedicated coprocessors to the embedded core; (3) a systematic memoryestimation and optimization technique to reduce the memory needs of the feature extraction process for embedded devices; (4) a more secure matching algorithm based on the local structure.

This paper is organized as follows. Section 2 reviews some related work. An overview of our proposed system is presented in Section 3. Then the algorithm and speed optimizations for feature extraction are discussed in Section 4 and the memory management in Section 5. In Section 6 we propose our secure matching technique. Finally we conclude this paper in Section 7 with the main contribution of our work.

\section{RELATED WORK}

Lots of research has been performed for the minutiae-based fingerprint matching. Some of them use the local structure of the minutiae to describe the characteristics of the minutiae set [4]. The alignment-based matching algorithms make use of the shape of the ridge connected to the minutiae [5]. Some other researches combine the local and global structures $[6,7]$. The local structure is used to find the correspondences of two minutiae sets and increase the reliability of the global matching. The global structure reliably determines the uniqueness of a fingerprint. The approach in [8] is similar to our work. However, we propose a new definition of the local structure of a minutia, which is proven efficient for low quality input fingerprints.

As new processors continuously improve the performance of embedded systems, the processor-memory gap widens and memory represents a major bottleneck in terms of speed, area, and power for many applications [9]. Memory-estimation techniques at the system level are used to guide the embedded system designer in choosing the best solution. In data dominated applications, summing up the sizes of all the arrays is the most straightforward way to get an upper bound of the memory requirement. However, "inplace" problem [10] introduces a huge overestimate. In [11], the internal in-place mapping is taken into consideration and the total storage requirement is the sum of the requirements for each array. In [12], the data dependency relations in the code are used to find the number of array elements produced or consumed by each assignment, from which a memory trace of upper and lower bounding rectangle, as a function of time, is found. In [13], a methodology based on live variable analysis and integer-point counting is described. The method introduced in this paper takes both the program size and the data size into consideration and provides an efficient way to reduce the memory requirements for embedded systems at the system level using the information gathered from runtime simulation.

For efficient fingerprint authentication system design on an embedded platform, recent researches have introduced coprocessor enhancements by a generic set of custom instruction extensions to an embedded processor instruction set architecture [14]. Besides the hardware/software codesign optimization, we also proposed software-level accelerate techniques in this paper.

\section{SYSTEM OVERVIEW}

In a traditional distributed system involving resource-limited embedded devices, usually the system partitioning is only based on distributing the computations between the embedded device and a main server for lowering the overall energy consumption. However, our proposed system requires a partitioning technique that also takes the security into consideration. Therefore, we need to perform the complete biometrics processing locally on the embedded device instead of offloading them to the server or the card reader. The proposed fingerprint verification system consists of four basic subsystems: data collection, minutiae extraction, matching, and communication. The first three take care of the biometric processing and matching, while the communication part allows the transmission of the result, a yes/no signal, 


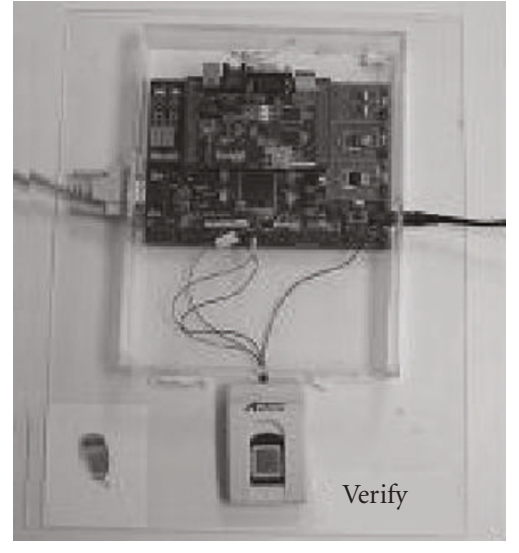

(a)

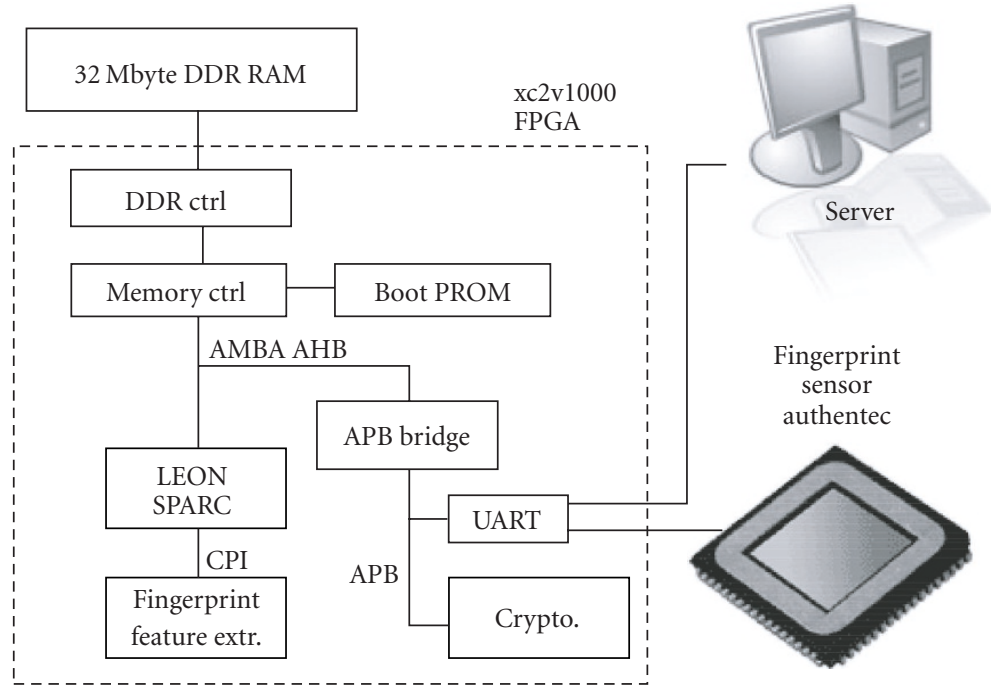

(b)

Figure 1: (a) FPGA board setup for demonstration; (b) prototype architecture.

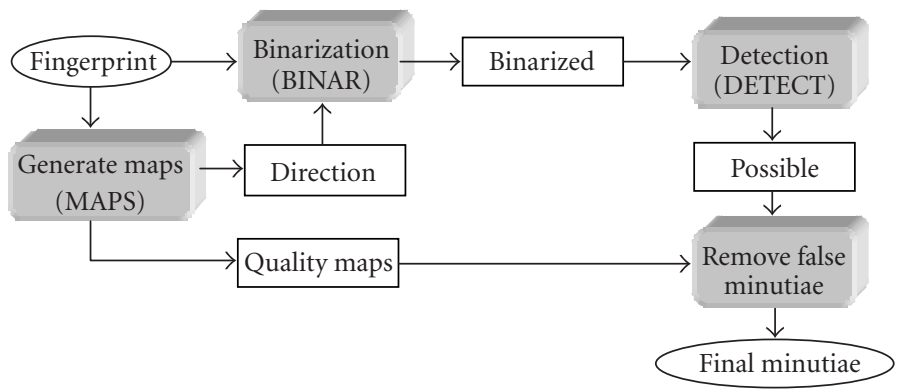

FIgURE 2: NIST minutiae extraction flow.

to the server. By doing this, the sensitive biometric data is confined to the embedded device and the only information transmitted is the final binary result, which is nonsensitive.

The hardware platform to demonstrate our system consists of a LEON-2 processor embedded in the Xilinx FPGA (Virtex-II), DDR SDRAM, and an Authentec AF-2 CMOS imaging fingerprint sensor. LEON-2 is a synthesizable VHDL model of a 32-bit processor compliant with SPARC V8 architecture. The model is highly configurable, and particularly suitable for system-on-chip (SOC) designs [15]. The demonstration setup and the architecture are shown in Figure 1. The fingerprint sensor is connected via the serial link to the FPGA board. The FPGA contains the soft LEON-2 SPARC core and two acceleration units, one for minutiae processing (DFT) and one for encryption purposes (AES).

To verify the fingerprint match algorithm, we apply our system to a subset of the FVC2000 fingerprint database [16]. In order to evaluate a realistic system performance, we have also constructed a new database using the Authentec AF-2 CMOS imaging sensor [17], which is a part of our fingerprint verification system. Ten live-scan fingerprint samples per finger from 10 different thumbs are captured, forming a test bench having a total of 100 fingerprint images.

\section{FEATURE EXTRACTION}

The feature-extraction step is the most computationintensive step. Its optimization to fit on an embedded device consists of several steps. The first step is the optimization of the algorithm itself to reduce the number of operations. The second step consists of identifying the computation bottlenecks and designing acceleration units for it. The third step consists of the memory optimization.

\subsection{Minutiae extraction algorithm}

The start point of the algorithm for extracting the minutiae of a fingerprint is taken from the NIST Fingerprint Image Software [18]. The basic steps are shown in Figure 2.

The fundamental step in the minutiae extraction process is deriving a directional ridge flow map to represent the orientation of the ridge structure (MAPS). To locally analyze 


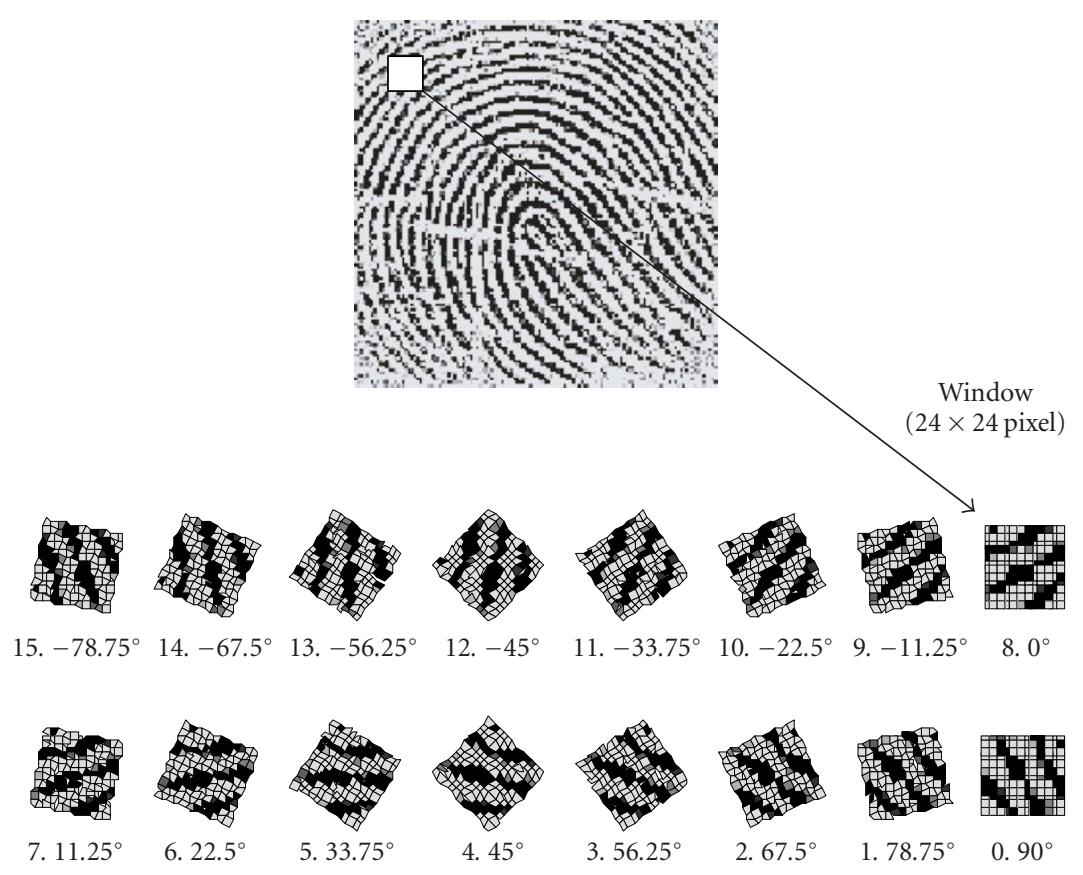

Figure 3: An example case of the window rotation.

the fingerprint, the image is divided into a grid of $8 \times 8$ pixel blocks with a larger surrounding $24 \times 24$ pixel window. For each block, the surrounding window is rotated incrementally and a discrete fourier Transform (DFT) analysis is conducted at each orientation. The number of orientations is set to 16 . Within an orientation, the pixels along each rotated row of the window are summed together, forming 16 vectors of row sums (see Figure 3). Each vector of row sums is convolved with 4 waveforms of increasing frequencies, producing resonance coefficients that represent how well the vector fits the specific waveform. The dominant ridge flow direction for the block is determined by the orientation with the maximum waveform resonance. Also the image quality is analyzed. The blocks, for which it is difficult to accurately determine the ridge flow, are marked, indicating that the minutiae detected within those blocks are less reliable.

Each pixel is assigned a binary value based on the ridge flow direction associated with the block to which the pixel belongs (BINAR). A $7 \times 9$ pixel grid is defined centered at the pixel. The angle of the grid row is set parallel to the local ridge flow direction. Then the center row sum and the average row sum are compared. If the center row sum is less than the average intensity, the center pixel is set to black; otherwise, it is set to white. Following the binarization, the detection step methodically scans the binary image of a fingerprint, identifying the localized pixel patterns that indicate the ending or bifurcation of a ridge (DETECT). Since the scanning technique is conservative to minimize the chance of missing true minutiae, the minutiae candidates pointed out by performing these steps need further refinement stages. Typical types of sources for the false minutiae include: (1) islands, lakes, and holes in the binarized image; (2) nonreliable minutiae in regions of poor image quality; (3) side minutiae, hooks, overlaps, minutiae that are too wide, and so forth. Considering these problems, several steps are performed to remove the false minutiae from the candidates list.

\subsection{High-speed accelerator}

Implementing the fingerprint verification module on an embedded device requires not only accuracy, but also highspeed and low power consumption. In this paper, we investigate both software and hardware optimization techniques to achieve this goal.

Software optimization aims at reducing the cycle count of the whole process. To get better performance, the first step is to find out the bottlenecks of the system. For this purpose, the TSIM SPARC simulator is used to profile the C code [15]. Simulation shows that the minutiae extraction process takes most ( 99\%) of the execution time. Therefore, we will focus on the speed optimization of this module. Figure 4(a) shows the profiling result of the minutiae extraction process. The execution time of the image binarization and the minutiae detection are $11 \%$ and $12 \%$ of the total, respectively, and they are not considered the system bottlenecks. However, the direction map deriving step (MAPS) occupies $74 \%$ of the total execution time. Therefore, the detailed algorithm for it is investigated further. Figure 4(b) shows the instruction-level profiling of the MAPS. The numbers of instructions for multiply (Mult) and addition (Add) sum up to $56 \%$ of the total MAPS processing due to the repetitive DFT calculations for creating the direction map. Based on 


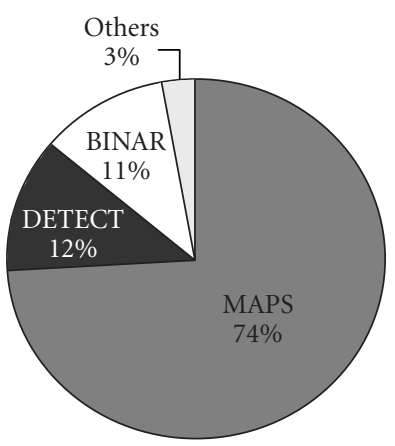

(a)

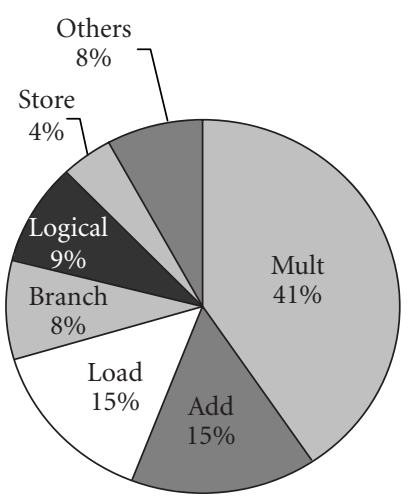

(b)

Figure 4: (a) Profiling of the execution time for the minutiae extraction; (b) instruction-level profiling of MAPS.

the profiling results, software optimization and hardware acceleration are considered for the DFT calculations in the directional map-deriving step.

\section{(1) Software optimization for the minutiae extraction}

Observing the directional map of a fingerprint, we find that the neighboring blocks tend to have similar directions due to the continuousness of the ridge flow. An example is shown in Figure 5. This characteristic can be used to significantly reduce the number of DFT calculations. For instance, the first direction data, upper left in Figure 5, is calculated using the same method as the original approach. After that, when deciding the direction of the block right next to it, instead of beginning with $\theta=0$, the DFTs for $\theta=4,5,6$ are first calculated because the result is most likely to be $\theta=5$. Generally, for each $\theta$, the pixels along each rotated row of the window are summed together forming a vector of 24 row sums $(\operatorname{row} \operatorname{sum}(i, \theta), i=0,1,2, \ldots, 23)$. Each vector of row sums is convolved with several waveforms. Discrete values for the sine and cosine functions at different frequencies $(\varphi)$ are computed for each unit along the vector. The row sums in a vector are then multiplied to their corresponding discrete sine values, and the results are accumulated and squared. The same computation is done between the row sums in the vector and their corresponding discrete cosine values.

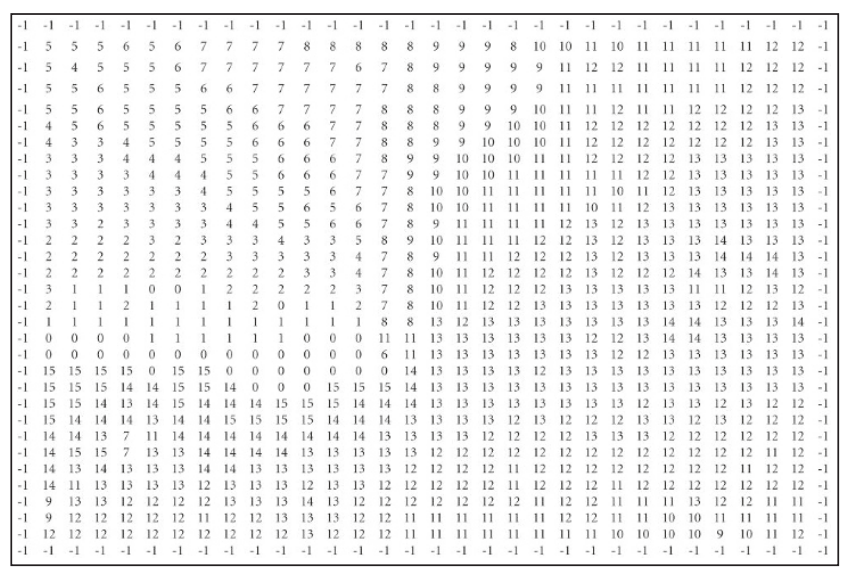

Figure 5: Example of direction map. “-1" means no direction because of the zero-padding in the image.

The squared sine component is then added to the squared cosine component, producing a resonance coefficient that represents how well the vector fits the specific waveform. The resonance coefficient is described as

$$
\begin{aligned}
E_{\text {Total }}(\theta) & =\sum_{\varphi}\left(A^{2}(\varphi, \theta)+B^{2}(\varphi, \theta)\right), \\
A(\varphi, \theta) & =\sum_{i=0}^{23} \operatorname{row} \operatorname{sum}(i, \theta) \bullet \sin \frac{\varphi \cdot i \cdot \pi}{16}, \\
B(\varphi, \theta) & =\sum_{i=0}^{23} \operatorname{row} \operatorname{sum}(i, \theta) \bullet \cos \frac{\varphi \cdot i \cdot \pi}{16} .
\end{aligned}
$$

For instance, if for $\theta=5$ the total energy is greater than both its neighbors $(\theta=4,6)$ as well as a threshold value $\left(E_{\mathrm{TH}}\right)$, the direction of $\theta=5$ is considered correct. Otherwise, $\theta$ is incremented or decremented until the total energy for it peaks with a value greater than $E_{\mathrm{TH}}$. In other words, if the three conditions in (2) are met, the direction of a block is determined. It is noted that the sine and cosine values are left-shifted by 16 bits for fixed-point refinement. The execution speed as well as the matching error rate is measured when $E_{\mathrm{TH}}$ is changed from $1.0 \times 10^{7}$ to $3.5 \times 10^{7}$. The experimental result shows that when $E_{\mathrm{TH}}$ is larger than $2.0 \times 10^{7}$, the error rate is within an acceptable range:

$$
\begin{gathered}
E_{\text {Total }}(\theta)>E_{\text {Total }}(\theta-1) \quad[\text { when } \theta=0, \theta-1=15], \\
E_{\text {Total }}(\theta)>E_{\text {Total }}(\theta+1) \quad[\text { when } \theta=15, \theta+1=0], \\
E_{\text {Total }}(\theta)>E_{\mathrm{TH}} .
\end{gathered}
$$

\section{(2) DFT accelerator for the minutiae extraction}

Software optimizations reduce the number of DFT calculations and result in a significant speedup of the minutiae extraction process. However, there are still a large number of DFT calculations, even if $E_{\mathrm{TH}}$ is set to a proper value. Therefore, DFT hardware acceleration is needed in addition to software optimization. A DFT coprocessor is designed to 


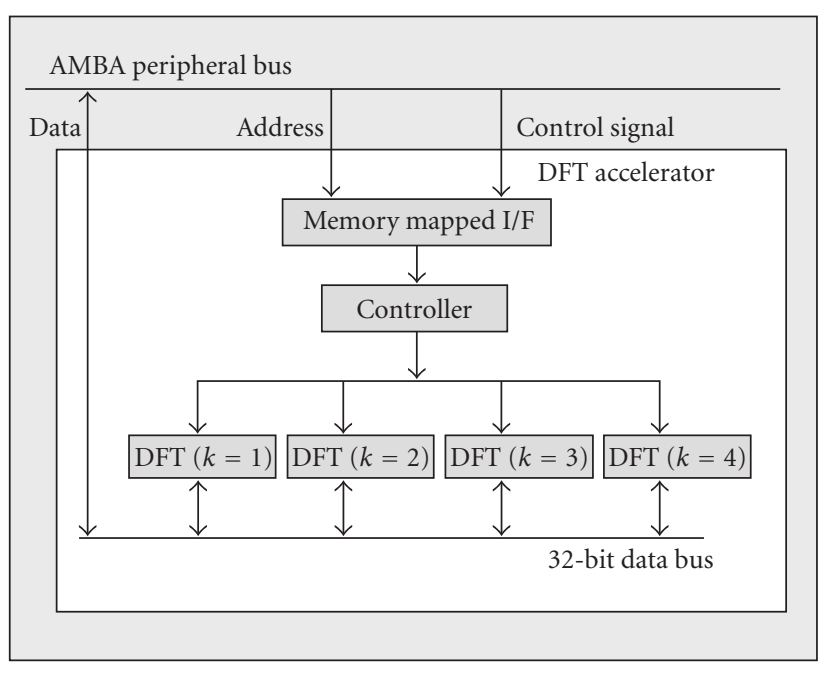

FIGURE 6: Block diagram for the memory-mapped DFT accelerator.

implement four parallel one-dimensional 24-point DFTs on four different discrete sample frequencies (see Figure 6).

The coprocessor is memory-mapped and two memory locations are used between the CPU and the coprocessor for the instructions and the data, respectively. The 16 row sum vectors are sent to the coprocessor and the sine and cosine accumulate results are retrieved. By performing this, the control flow and the data flow of the DFT algorithm are separated into the embedded LEON-2 processor and the DFT coprocessor, respectively [19]. This coprocessor design has been done with the design environment GEZEL [20]. With the GEZEL environment, a cosimulation is setup between the software running on the embedded core and the hardware acceleration units. GEZEL facilitates the codevelopment of hardware accelerator units and software optimization on the embedded platform. The area cost for the DFT coprocessor is 2844 LUTs and whole system requires 7700 LUTs after place and route. The energy calculation part is not included because it needs a square operation of 16-bit data, which requires a general multiplier. As a result, the execution time of the minutiae extraction is reduced to about 4 seconds from originally 9 seconds resulting from the fixed-point implementation on the $50 \mathrm{MHz}$ LEON-2 processor, as shown in Figure 7(a). This system speed is among the top results in the light category of FVC2004 [21]. In the meantime, the energy consumption is reduced from $5.187 \mathrm{~mJ}$ to $2.500 \mathrm{~mJ}$ in case of $E_{\mathrm{TH}}=2.7 \times 10^{7}$ as presented in Figure 7(b). In order to obtain the energy estimation, the power is simulated using Xilinx's Xpower and we get the total system cycle number from cycle true simulation with GEZEL.

\section{MEMORY OPTIMIZATION}

As mentioned before, in a fingerprint verification system, the major computational bottleneck is the fingerprint minutiae extraction. Like many other image processing algorithms, it is array-dominated. Therefore, apart from optimizations

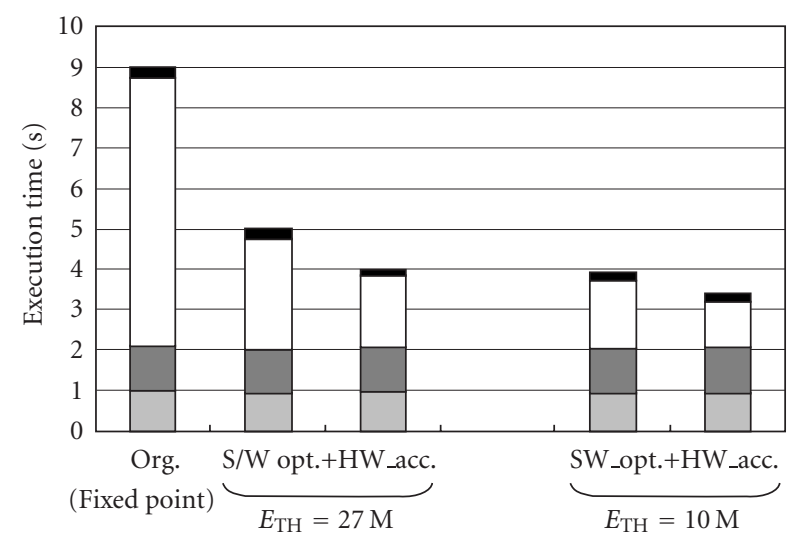

OTHERS

MAPS

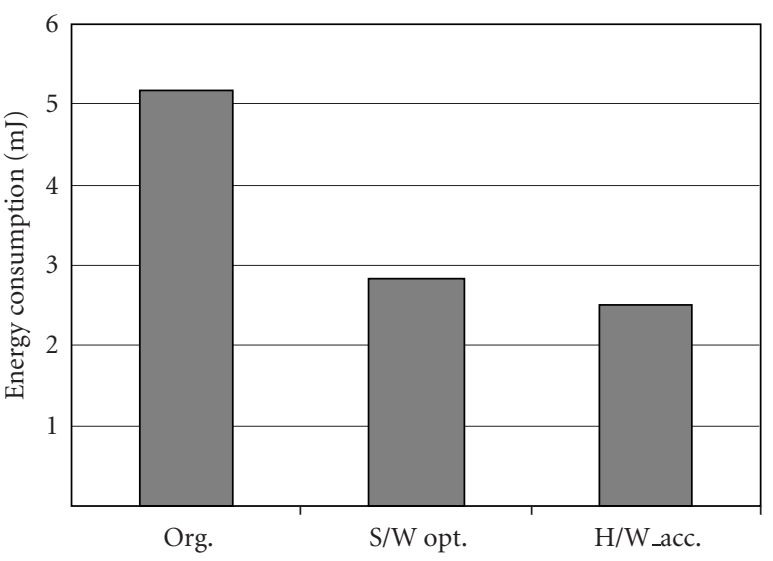

(b)

FIgURE 7: (a) Reduction of the execution time for the minutiae extraction; (b) reduction of the energy consumption for the minutiae extraction $\left(E_{\mathrm{TH}}=2.7 \times 10^{7}\right)$.

for high-speed calculation, memory management is also necessary. In this section, we will introduce a memory analysis method. Several memory optimization techniques are implemented based on the analysis results.

\subsection{Memory analysis methodology}

When a program is running, the memory space is divided into two parts: a program segment and a data segment. The data segment includes a heap and a stack. The heap starts from the bottom of the program segment and increases when the latest reserved memory block is beyond its range. Whenever there is dynamic memory allocation, a block of memory is reserved for later use. When a memory free happens, the specific memory block is returned to the memory pool. On the other hand, the stack pointer position changes when a function call is executed or returned. Generally, the stack and the heap grow and shrink in opposite direction. A collision 


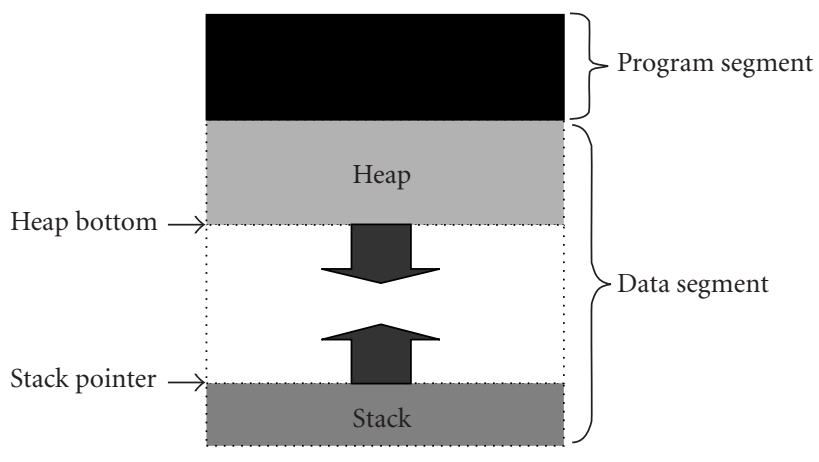

FIGURE 8: Memory partitioning during the program running time.

of the stack and the heap implies a fatal error state. At any particular moment, the memory usage of the system is determined by the sum of the size of the program, the heap, and the stack as shown in Figure 8.

By inserting the memory trace agents in the program where memory usage changes can happen, we get the position of the heap bottom and the stack pointer dynamically during the program run time. Taking the program size into consideration, a dynamic memory usage trace map is generated. From the trace map, we can get information about the dynamic memory requirement as well as the memory bottleneck of the application.

\subsection{Baseline result for the minutiae detection}

Applying the methodology described in the previous section to the baseline minutiae extraction algorithm, a memory trace map is obtained (see Figure 9(a), where the $x$ axis shows the number of memory change points). The peak memory usage of the system is 1.572 Kbytes, including 325 Kbytes of program segment memory and 1.247 Kbytes of data segment memory. For most portable embedded systems, a memory size beyond 1 Mbytes is too expensive. In order to reduce the memory requirement for this application, we try to minimize the program size as well as the running time memory usage based on the information obtained from the memory trace map.

\subsection{Memory optimization}

\section{(1) Architecture optimization}

The NIST starting point program, as is the case for most fingerprint extraction algorithms, is floating-point based, while the LEON-2 processor, as most low power embedded processor cores, only supports fixed-point computation. Therefore, we perform a fixed-point refinement optimization by replacing all the floating-point variables with 32-bit long integer ones. From the memory trace map (see Figure 9(b)) of the fixed-point refined program, we notice that both the program segment size and data segment size decrease. This is because, on the one hand, the fixed-point refinement removes the floating-point calculation-related libraries; on the other hand, the size of the elements of most arrays are modified from the 8-byte "double" type to the 4-byte "int" type, which reduces the storage memory by half. In total, the memory requirement for a fixed-point refined program is 1.267 Kbytes.

\section{(2) In-place optimization}

The memory trace maps in Figures 9(a) and 9(b) show that there is a major jump which introduces most of the memory usage in a very short period. Our idea for reducing the data segment memory is first finding out where the jump happens, then analyzing the algorithm to figure out the reason for the major memory usage and implementing memory management techniques to remove or lower the jump.

Detailed investigation of the minutiae extraction algorithm shows that the biggest jump happens when a routine named "pixelize_map" is called. The functionality of this routine is to convert the block-based maps for direction, lowflow flag, and high-curve flag into pixel-based ones. For each pixelized map, $262.144(256 \times 256 \times 4)$ bytes of memory are required since for each pixel, one 32 -bit integer is used to present each value. This results in the jump in the memory trace map.

The dimensions for the three maps are exactly the same. Moreover, the values in direction_map vary from 0 to 32 and low_flow_map and high_curve_map consist of only 0 and 1 . Therefore taking one corresponding element from each map, only 6 bits are required per pixel ( 4 bits for direction_map, 1 bit for low_flow_map, and 1 bit for high_curve_map). It is possible to merge these three different maps into one map since we can combine the three elements (one from each map) in one 32-bit integer. In compiler terminology, this operation is called loop merging [22]. By implementing this compression, the peak memory requirement becomes 744 Kbytes (see Figure 9(c)). The data segment memory decreases by 590 Kbytes compared to the previous result, while the program segment size slightly increases by 47 Kbytes due to the additional calculations, which are needed for the compression and decompression of the pixelized maps.

\section{(3) Online calculation}

As shown in Figure 9(c), the memory requirement bottleneck is still in the pixelize_map routine. Further optimization can be implemented by reordering the sequence of calculations [22]. Instead of generating the complete pixelized maps, storing them and then using them, we adopt a running time calculation for the map value of each pixel. It is a form of "just-in-time" calculations: a map element is generated by the program only when it is referred to during run time. This technique removes the major memory usage jump in the memory trace map, but it does require an analysis of the relative creation time and consumption time of the map values. A minimum memory size is obtained when the creation is just before the consumption [23]. The drawback of it is that the pixel index needs to be calculated each time it is referred. However, using this online calculation, the time consuming routine for generating the pixelized maps is 


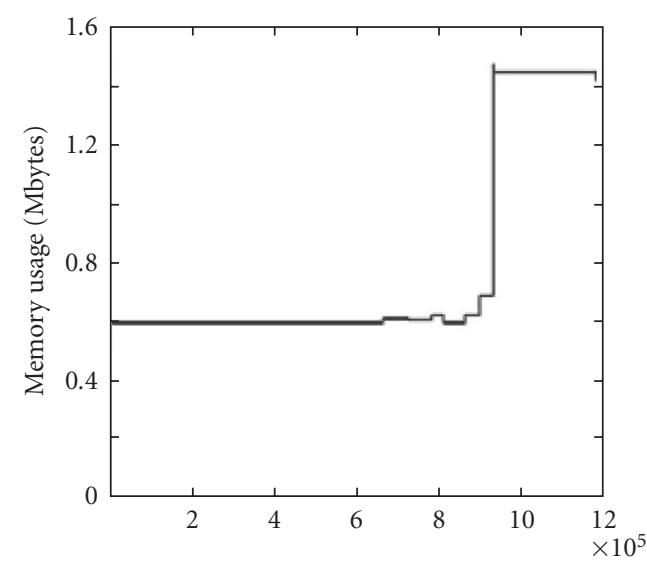

(a)

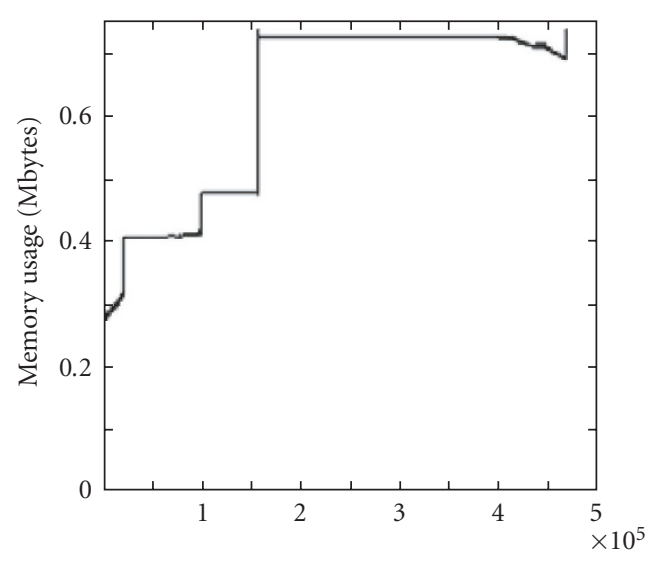

(c)

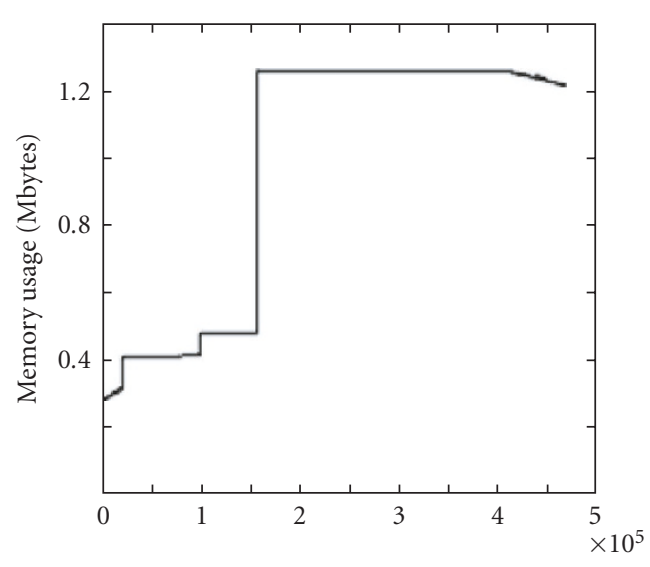

(b)

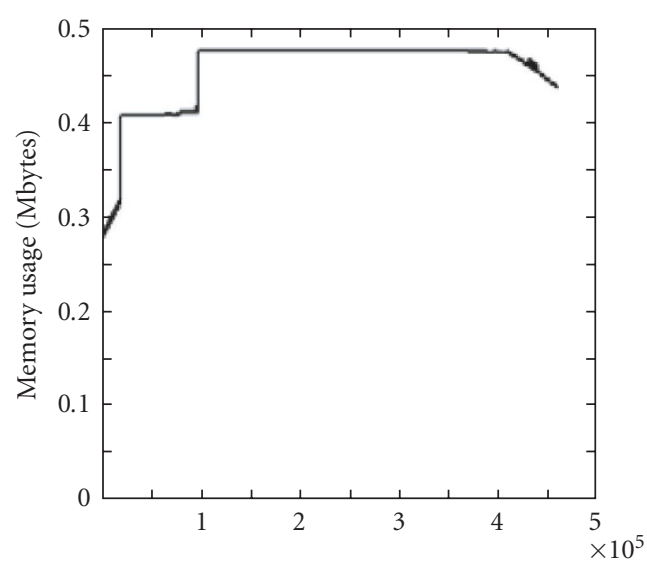

(d)

FIGURE 9: Memory trace maps for (a) baseline program, (b) architecture optimization, (c) in-place optimized, (d) online calculation.

skipped, thus it is found that this technique will save memory with no cost of speed. The result of this method is shown as Figure 9(d). Comparison of the results shows that both the program segment size and the data segment size decrease. The total memory requirement is 483 Kbytes, which outperforms all the algorithms in the light category of FVC2004 [21]. Figure 10 shows the memory reduction for the optimization techniques introduced before.

\section{MATCHING}

The matching step compares the candidate fingerprint against the stored template. It uses the minutiae obtained from the previous steps to perform this comparison. A novel more secure matching algorithm is proposed in our system. Unlike most of the existing techniques, this algorithm is only based on the local neighborhood structure of the fingerprint minutiae. There are two main reasons we proposed this matching technique. First, a pure local structure does not rely on any global information; therefore no calculation is needed for alignment. This makes the algorithm very efficient in terms of speed. Secondly, this algorithm will increase the system security since the global picture of the fingerprint cannot be easily obtained even when the stored templates are disclosed.

\subsection{Algorithm}

From the result of the minutiae extraction step, information such as the $x, y$ coordinates and the local ridge direction is available for each minutia. As mentioned before, direct storage of the minutiae set could lead to disclosure of the biometric information. To enhance the security of the system, our newly proposed technique is based on a derived local structure. Generally, given one minutia $M$, we define a new local structure of it which is described as a feature vector:

$$
L_{M}=\left\{d_{1}, d_{2}, \ldots, d_{N}, \varphi_{1}, \varphi_{2}, \ldots, \varphi_{N}, \vartheta_{1}, \vartheta_{2}, \ldots, \vartheta_{N}, \Psi\right\}
$$

where $N$ is the number of neighbors taken into consideration during matching. $\Psi$ is the local ridge direction of the minutia $M \cdot d_{n}(n=1,2, \ldots, N)$ describes the distance between the selected minutia $M$ and its $n$th nearest neighbor, $\varphi_{n}(n=1,2, \ldots, N)$ is the related radial angle between $M$ and its $n$th nearest neighbor, and $\theta_{n}(n=1,2, \ldots, N)$ represents 


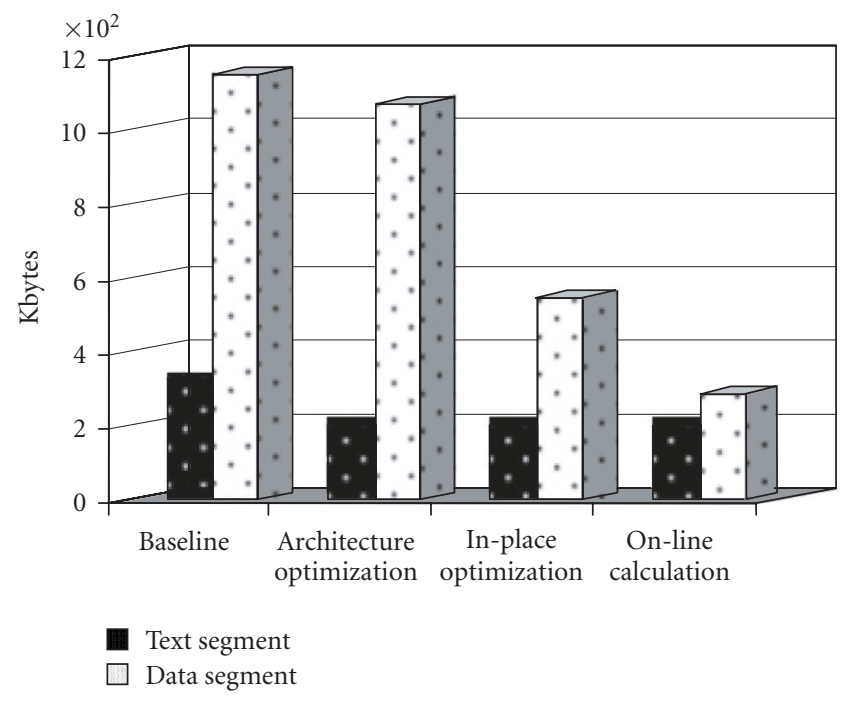

FIGURE 10: Memory-reduction techniques for minutiae extraction.

the related position angle of the $n$th nearest neighbor. One example for $N=2$ is shown in Figure 11, describing the local structure of a minutia with its two nearest neighbors. All the elements in the local structure can be calculated from the information obtained from the minutiae extraction following (4):

$$
\begin{gathered}
d_{n}=\sqrt{\left(x_{n}-x_{0}\right)^{2}+\left(y_{n}-x_{0}\right)^{2}}, \\
\varphi_{n}=\operatorname{diff}\left(\Psi_{n}, \Psi\right), \\
\vartheta_{n}=\operatorname{diff}\left(\arctan \left(\frac{y_{n}-y_{0}}{x_{n}-x_{0}}\right), \Psi\right), \quad n=1,2, \ldots, N .
\end{gathered}
$$

The function $\operatorname{diff}(\cdot)$ calculates the difference of two angles and ports the result to the range $[0,2 \pi)$. When two minutiae are compared, the relative position and angles of their $N$ nearest neighbor minutiae are examined. We can rewrite (3) to obtain an alternative form of the local feature vector. Assume one minutia $M$ in the input fingerprint is

$$
L_{M}=\left\{\left\{d_{1}, \varphi_{1}, \vartheta_{1}\right\},\left\{d_{2}, \varphi_{2}, \vartheta_{2}\right\}, \ldots,\left\{d_{N}, \varphi_{N}, \vartheta_{N}\right\}, \Psi\right\}
$$

and one minutia $M^{\prime}$ in the stored template is

$$
L_{M^{\prime}}=\left\{\left\{d_{1}^{\prime}, \varphi_{1}^{\prime}, \vartheta_{1}^{\prime}\right\},\left\{d_{2}^{\prime}, \varphi_{2}^{\prime}, \vartheta_{2}^{\prime}\right\}, \ldots,\left\{d_{N}^{\prime}, \varphi_{N}^{\prime}, \vartheta_{N}^{\prime}\right\}, \Psi\right\} .
$$

The proposed matching algorithm calculates how similar the neighborhood of one minutia in the input fingerprint is to that of one in the stored template. If it is similar enough, these two minutiae are taken as a "matched" minutiae pair. After each minutia pair is compared, the total number of "matched" minutiae pairs is used to calculate the final matching score.

To decide whether or not $M$ and $M^{\prime}$ are a matched minutiae pair, a small four-dimensional range box is set for $(d, \varphi, \vartheta, \Psi)$, respectively: $\left\{\Delta_{d}, \Delta_{\varphi}, \Delta_{\vartheta}, \Delta_{\Psi}\right\}$. The first step is to check the local ridge directions of the two minutiae. If $\left|\Psi-\Psi^{\prime}\right|>\Delta_{\Psi}, M$ and $M^{\prime}$ are not matched. Therefore the

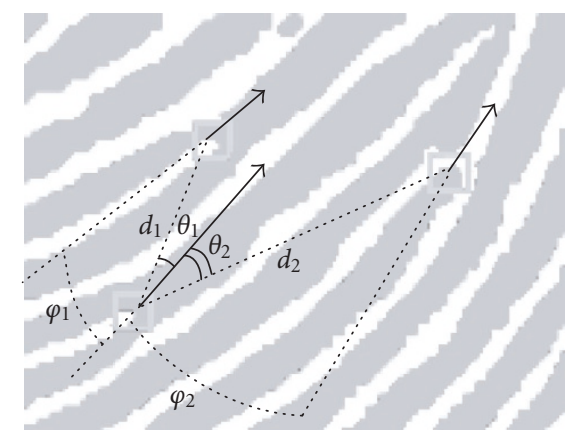

FIgURE 11: Local structure of a minutia $(N=2)$.

matcher searches for another minutiae pair. Otherwise, the matcher continues to investigate the neighbor minutiae according to the neighborhood condition described in (7):

$$
\begin{gathered}
\left|d_{i}-d_{j}^{\prime}\right| \Longleftarrow \Delta_{d}, \quad\left|\varphi_{i}-\varphi_{j}^{\prime}\right| \Longleftarrow \Delta_{\varphi}, \\
\left|\vartheta_{i}-\vartheta_{j}^{\prime}\right| \Longleftarrow \Delta_{\vartheta} .
\end{gathered}
$$

If the conditions in (7) are all satisfied, the $i$ th neighbor of the input minutia $M$ and the $j$ th neighbor of the template minutia $M^{\prime}$ are considered "marked." After a thorough check of all the neighbor minutiae of $M$ and $M^{\prime}$, the number of marked neighbor pairs is accumulated as $A$. If this number is above a specific threshold $\mathrm{TH}_{A}$, the minutiae $M$ and $M^{\prime}$ are considered as a matched minutiae pair. The threshold is set according to experimental results, which we will discuss later. Following this procedure, a comparison of all the minutiae in the input and template fingerprints results in the total number of matched minutiae pairs, $B$. Assuming that the numbers of the minutiae of input and template fingerprints are $\mathrm{NUM}_{\text {input }}$ and $\mathrm{NUM}_{\text {temp }}$, respectively, the final matching score is calculated as

$$
\text { score }=\frac{B}{\max \left(\mathrm{NUM}_{\mathrm{input}}, \mathrm{NUM}_{\mathrm{temp}}\right)} .
$$

Two fingerprints will be verified as from the same finger if their matching score is higher than a certain threshold.

According to the descriptions of the matching algorithm, the template, which is stored in the embedded device, consists only of the local relationship between each minutia and its neighbors. Unlike other minutiae-based fingerprint verification systems, there is no global information about the whole fingerprint stored. Therefore, even if the stored template is compromised, it cannot be used to reconstruct the original minutiae set of the fingerprint.

\subsection{Definition of neighborhood structure}

Our proposed matching method is based on the local structure of the minutiae. The selection of the number of neighbors is very important for the system performance. If the number is too small, which indicates a relative loose matching condition, some nonmatched minutiae pairs, which are somehow similar, are very likely to satisfy the matching conditions. This may lead to a high false accept rate (FAR). On 
Table 1: Possibility to achieve baseline accuracy for different localstructure definitions and thresholds.

\begin{tabular}{|c|c|c|c|c|c|}
\hline & \multicolumn{4}{|c|}{ Number of neighbors in local structure } \\
\hline & & 4 & 5 & 6 & 7 \\
\hline \multirow{5}{*}{$\begin{array}{l}\text { Thresholds } \\
\left(\mathrm{TH}_{A}\right)\end{array}$} & 2 & No & No & No & No \\
\hline & 3 & No & Yes & Yes & No \\
\hline & 4 & - & No & No & No \\
\hline & 5 & - & - & No & No \\
\hline & 6 & - & - & - & No \\
\hline
\end{tabular}

the contrary, if the neighbor number is set too large, the matching condition becomes very strict. Many matched pairs may fail because the fingerprint image is sometimes incomplete and the minutiae detection is not very precise. This may result in a high false reject rate (FRR). In order to choose the proper neighborhood structure which could achieve reasonable FRR and FAR, experiments are performed for different local structure definitions, where the number of neighbor minutiae taken into account varies from 4 to 7 . For each local structure definition, matching accuracy for different marked neighbor pair thresholds is investigated. In this work we use $1 \%$ FRR and $0.01 \%$ FAR as the baseline accuracy needed for modern biometric systems [24], Table 1 presents the possibility to reach this standard for different cases.

From Table 1 it is found that the matching algorithm based on minutiae structure including 5 or 6 neighbors can achieve desirable accuracy with the marked pair threshold of 3. Further results are shown in Figure 12 for these two cases. The $x$-axis is the FRR and the $y$-axis shows the FAR. After analyzing the result, we define the number of neighbors as 6 and the marked neighbor pair threshold $\mathrm{TH}_{A}$ is set to 3 in our work. By selecting this local structure, we achieve an FRR of $1 \%$ and an FAR of less than $0.1 \%{ }^{1}$

Also we compared the template size of our matching algorithm with others. For a typical case, $0 \leq d_{i} \leq 256$, $0 \leq \varphi_{i}, \theta_{i}, \Psi \leq 32$, the average template size for our algorithm is around $0.5 \mathrm{kbytes}$, which is comparable to the template size in the light category of FVC2004 [21].

\section{CONCLUSION}

In this article, we demonstrate that it is feasible and it can be done to implement a complete fingerprint authentication system on a $50 \mathrm{MHz}$ embedded platform. To address the security problem for biometric authentication systems, we propose a novel secure fingerprint verification technique, within which the matching algorithm is based on a well-defined local neighborhood structure of the minutiae.

In order to speed up the fingerprint image processing, a set of software and hardware optimizations methods are applied, gaining a $65 \%$ execution time reduction with less than half the energy consumption. A memory analysis method is

\footnotetext{
${ }^{1}$ In the simulation of our database, there is no false accept error.
}

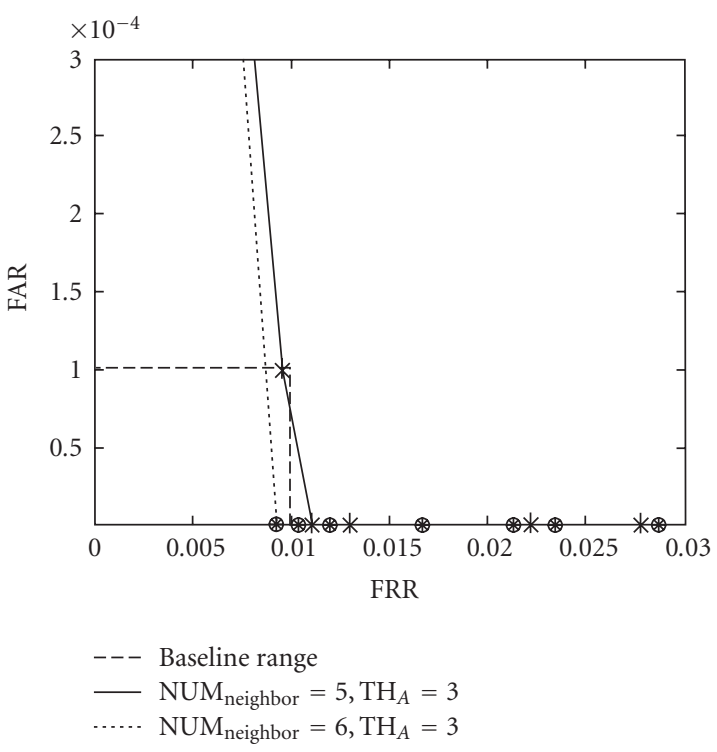

FIGURE 12: False reject rate (FRR) and false accept rate (FAR) for different selections of local structure.

introduced to trace the program memory usage during run time. Based on the analysis results, memory-optimization techniques and code transformations are implemented and $67 \%$ memory storage requirement reduction is achieved. This results in an implementation that ranks in the top of the light category, with an execution time of less than 4 seconds on $50 \mathrm{MHz}$ platform, an energy estimate on an FPGA of $2500 \mathrm{~mJ}$, and a memory size, which is the smallest in the light category of FVC2004. This work successfully ports the complete fingerprint processing, which is usually done on a central server, to a resource constraint embedded device.

\section{ACKNOWLEDGMENTS}

This work was supported by the NSF, account no. CCR0098361, the Langlois Foundation, and UC MICRO. The authors would like to thank all the teammates in the ThumbPod project $[25,26]$. We also thank Gaisler Research for providing the LEON-2 SPARC core and for support in setting up the simulation environment [15].

\section{REFERENCES}

[1] Y. Gil, D. Moon, S. Pan, and Y. Chung, "Fingerprint verification system involving smart card," in Proceedings of the International Conference on Information Security and Cryptology, vol. 2587 of LNCS, pp. 510-524, Seoul, Korea, November 2003.

[2] S. Prabhakar, S. Pankanti, and A. K. Jain, "Biometric recognition: security and privacy concerns," IEEE Security and Privacy Magazine, vol. 1, no. 2, pp. 33-42, 2003.

[3] V. Raghunathan, C. Schurgers, S. Park, and M. B. Srivastava, "Energy-aware wireless microsensor networks," IEEE Signal Processing Magazine, vol. 19, no. 2, pp. 40-50, 2002.

[4] A. K. Hrechak and J. A. Mchugh, "Automated fingerprint recognition using structural matching," Pattern Recognition, vol. 23, no. 8, pp. 893-904, 1990. 
[5] A. Jain, L. Hong, and R. Bolle, "On-line fingerprint verification," IEEE Transactions on Pattern Analysis and Machine Intelligence, vol. 19, no. 4, pp. 302-314, 1997.

[6] X. Jiang and W.-Y. Yau, "Fingerprint minutiae matching based on the local and global structures," in Proceedings of the International Conference on Pattern Recognition, pp. 6038-6041, Barcelona, Spain, September 2000.

[7] S. Yang, K. Sakiyama, and I. Verbauwhede, "A compact and efficient fingerprint verification system for secure embedded devices," in Proceedings of the 37th Asilomar Conference on Signal Systems, and Computers, pp. 2058-2062, Pacific Grove, Calif, USA, November 2003.

[8] A. Wahab, S. H. Chin, and E. C. Tan, "Novel approach to automated fingerprint recognition," IEE Proceedings - Vision, Image and Signal Processing, vol. 145, no. 3, pp. 160-166, 1998.

[9] P. Panda, F. Catthoor, N. Dutt, et al., "Data and memory optimization techniques for embedded systems," ACM Transactions on Design Automation of Electronic Systems, vol. 6, no. 2, pp. 149-206, 2001.

[10] I. Verbauwhede, F. Catthoor, J. Vandewalle, and H. De Man, "Background memory management for the synthesis of algebraic algorithms on multi-processor DSP chips," in Proceedings of the International Conference on VLSI (VLSI '89), pp. 209-218, Munich, Germany, August 1989.

[11] I. Verbauwhede, C. Scheers, and J. Rabaey, "Memory estimation for high level synthesis," in Proceedings of the 31st ACM/IEEE Design Automation Conference, pp. 143-148, San Diego, Calif, USA, June 1994.

[12] P. Grun, F. Balasa, and N. Dutt, "Memory size estimation for multimedia applications," in Proceedings of the 6th International Workshop on Hardware/Software Codesign, pp. 145-149, Seattle, Wash, USA, March 1998.

[13] Y. Zhao and S. Malik, "Exact memory size estimation for array computations without loop unrolling," in Proceedings of the 36th ACM/IEEE Design Automation Conference, pp. 811816, New Orleans, La, USA, June 1999.

[14] P. Gupta, S. Ravi, A. Raghunathan, and N. K. Jha, "Efficient fingerprint-based user authentication for embedded systems," in Proceedings of Design Automation Conference, pp. 228-233, Anaheim, Calif, USA, June 2005.

[15] http://www.gaisler.com.

[16] FVC2000: The First International Fingerprint Verification Competition, http://bias.csr.unibo.it/fvc2000/.

[17] http://www.authentec.com.

[18] M. D. Garris, C. I. Watson, R. M. McCabe, and C. L. Wilson, "User's Guide to NIST Fingerprint Image Software (NFIS)," NISTIR 6813, National Institute of Standards and Technology.

[19] P. Schaumont, K. Sakiyama, A. Hodjat, and I. Verbauwhede, "Embedded software integration for coarse-grain reconfigurable architectures," in IEEE 18th International Parallel and Distributed Processing Symposium (IPDPS '04), pp. 137-142, Santa Fe, NM, USA, April 2004.

[20] P. Schaumont and I. Verbauwhede, "Interactive cosimulation with partial evaluation," in Proceedings of Design Automation and Test in Europe (DATE '04), pp. 642-647, Paris, France, February 2004.

[21] FVC2004: The Third International Fingerprint Verification Competition, http://bias.csr.unibo.it/fvc2004/.

[22] K. Danckaert, F. Catthoor, and H. De Man, "A loop transformation approach for combined parallelization and data transfer and storage," in Proceedings of International Conference on Parallel and Distributed Processing Techniques and Applications (PDPTA '00), vol. 5, pp. 2591-2597, Las Vegas, Nev, USA, June 2000.
[23] F. Catthoor, K. Danckaert, S. Wuytack, and N. D. Dutt, "Code transformations for data transfer and storage exploration preprocessing in multimedia processors," IEEE Design \& Test of Computers, vol. 18, no. 3, pp. 70-82, 2001.

[24] R. J. Anderson, Security Engineering, A Guide to Building Dependable Distributed Systems, chapter 13, John Wiley \& Sons, New York, NY, USA, 2001.

[25] http://www.ThumbPod.com.

[26] D. Hwang, P. Schaumont, Y. Fan, et al., "Design flow for HW/SW acceleration transparency in the ThumbPod secure embedded system," in Proceedings of the 40th ACM/IEEE Design Automation Conference, pp. 60-65, Anaheim, Calif, USA, June 2003.

Shenglin Yang received her B.S. and M.S. degrees in electronics from Beijing University, Beijing, China, in 1998 and 2001, respectively. She is now pursuing her Ph.D. degree in electrical engineering at University of California, Los Angles. Her research interests include biometrics, pattern recognition, embedded implementation, and security system design. She is currently working on the biometric authentication system for embedded devices. She is a Student Member of IEEE.

Kazuo Sakiyama obtained the B.Eng. and M.Eng. degrees in electrical engineering from Osaka University, Japan, in 1994 and 1996, respectively. From 1996 to 2004, he was with the Semiconductor and IC Division of Hitachi, Ltd. (now Renesas Technology Corp.). During this time, he received the M.S. degree in electrical engineering from the University of California, Los Angeles. He is currently working on a Ph.D.

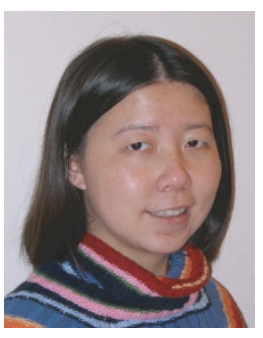
program at the Katholieke Universiteit Leuven, Belgium. His main research interest is efficient and secure embedded system architectures and design methodologies.

Ingrid Verbauwhede's interests include circuits, processor architectures, and design methodologies for real-time, embedded systems for applications such as security, cryptography, digital signal processing, and wireless applications. She received the Electrical Engineering degree in 1984 and the Ph.D. degree in applied sciences from the KULeuven, Leuven, Belgium, in 1991. She was a Lecturer and Visiting Research En-

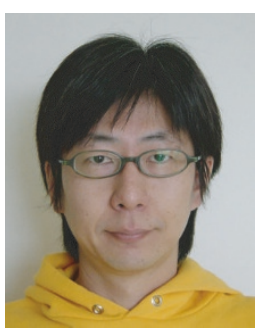
gineer at UC Berkeley from 1992 to 1994. From 1994 to 1998, she was a Principal Engineer first with TCSI and then with Atmel in Berkeley, Calif. She joined UCLA in 1998 as an Associate Professor and the KULeuven in 2003. At UCLA she ran the EMSEC, embedded security group. At KULeuven she is Codirector of the ESAT-COSIC research group. She was the General Chair of the IEEE International Symposium on Low Power Electronic Devices (ISLPED) in 2003. She is or was a member of several program committees, including DAC, ISSCC, DATE, CHES, ICASSP, SIPS, ASAP. She is the Design Community Chair on the 42nd and 43rd DAC Executive Community. She is a Senior Member of IEEE. 